\title{
Age-Related Hearing Loss Is Dominated by Damage to Inner Ear Sensory Cells, Not the Cellular Battery That Powers Them
}

\author{
${ }^{\circledR}$ Pei-zhe Wu, ${ }^{1,2,3}$ Jennifer T. O’Malley, ${ }^{1}$ Victor de Gruttola, ${ }^{4}$ and ${ }^{\circledR}$ M. Charles Liberman ${ }^{1,2}$ \\ ${ }^{1}$ Eaton-Peabody Laboratories, Massachusetts Eye and Ear, Boston, Massachusetts 02114, ${ }^{2}$ Department of Otolaryngology, Harvard Medical School, \\ Boston, Massachusetts 02115, ${ }^{3}$ Department of Otorhinolaryngology Head and Neck Surgery, First Affiliated Hospital, Sun Yat-sen University, \\ Guangzhou, 510080, China, and ${ }^{4}$ Department of Biostatistics, Harvard T.H. Chan School of Public Health, Boston, Massachusetts 02115
}

Age-related hearing loss arises from irreversible damage in the inner ear, where sound is transduced into electrical signals. Prior human studies suggested that sensory-cell loss is rarely the cause; correspondingly, animal work has implicated the stria vascularis, the cellular "battery" driving the amplification of sound by hair cell "motors." Here, quantitative microscopic analysis of hair cells, auditory nerve fibers, and strial tissues in 120 human inner ears obtained at autopsy, most of whom had recent audiograms in their medical records, shows that the degree of hearing loss is well predicted from the amount of hair cell loss and that inclusion of strial damage does not improve the prediction. Although many aging ears showed significant strial degeneration throughout the cochlea, our statistical models suggest that, by the time strial tissues are lost, hair cell death is so extensive that the loss of battery is no longer important to pure-tone thresholds and that audiogram slope is not diagnostic for strial degeneration. These data comprise the first quantitative survey of hair cell death in normal-aging human cochleas, and reveal unexpectedly severe hair cell loss in low-frequency cochlear regions, and dramatically greater loss in high-frequency regions than seen in any aging animal model. Comparison of normal-aging ears to an age-matched group with acoustic-overexposure history suggests that a lifetime of acoustic overexposure is to blame.

Key words: auditory; disorders of nervous system; sensory and motor system

Significance Statement

This report upends dogma about the causes of age-related hearing loss. Our analysis of over 120 autopsy specimens shows that inner-ear sensory cell loss can largely explain the audiometric patterns in aging, with minimal contribution from the stria vascularis, the "battery" that powers the inner ear, previously viewed as the major locus of age-related hearing dysfunction. Predicting inner ear damage from the audiogram is critical, now that clinical trials of therapeutics designed to regrow hair cells are underway. Our data also show that hair cell degeneration in aging humans is dramatically worse than that in aging animals, suggesting that the high-frequency hearing losses that define human presbycusis reflect avoidable contributions of chronic ear abuse to which aging animals are not exposed.

\section{Introduction}

Presbycusis is age-related hearing loss that cannot be explained by recognized otologic diseases, known ototoxins, or hereditary factors. Hearing loss decreases the quality of life and is a major contributor to social isolation and cognitive decline in the elderly

\section{Received Apr. 21, 2020; revised May 26, 2020; accepted May 27, 2020.}

Author contributions: P.Z.W. and M.C.L. designed research; P.Z.W. and J.T.O. performed research; P.Z.W. and V.d.G. analyzed data; P.Z.W. wrote the first draft of the paper; P.Z.W., J.T.O., and M.C.L. edited the paper; P.Z.W. and M.C.L. wrote the paper.

This work was supported by National Institute on Deafness and Other Communication Disorders Grant P50 DC 015857, and the Lauer Tinnitus Center at the Massachusetts Eye and Ear. The data that support the findings of this study are available in MATLAB format from the corresponding author upon reasonable request. The code that supports the findings of this study are available from the corresponding author upon reasonable request.
(Goman and Lin, 2018). Approximately 50\% of 85-year-olds have hearing loss; thus, the problem will grow as the population ages. The classic audiometric pattern in presbycusis shows a monotonic decrease of hearing sensitivity with increasing pitch of the test-frequency tones (Fig. 1D).

Presbycusis arises in the inner ear (Keithley, 2019; Tawfik et al., 2019; Fischer et al., 2020), where sound is transduced, by inner hair cells (IHCs), into electrical signals carried by auditory nerve fibers (ANFs) to the brain (Fig. $1 A, C$ ). The stria vascularis

The authors declare no competing financial interests.

Correspondence should be addressed to Pei-zhe Wu at Peizhe_Wu@meei.harvard.edu.

https://doi.org/10.1523/JNEUROSCI.0937-20.2020

Copyright $\odot 2020$ the authors 

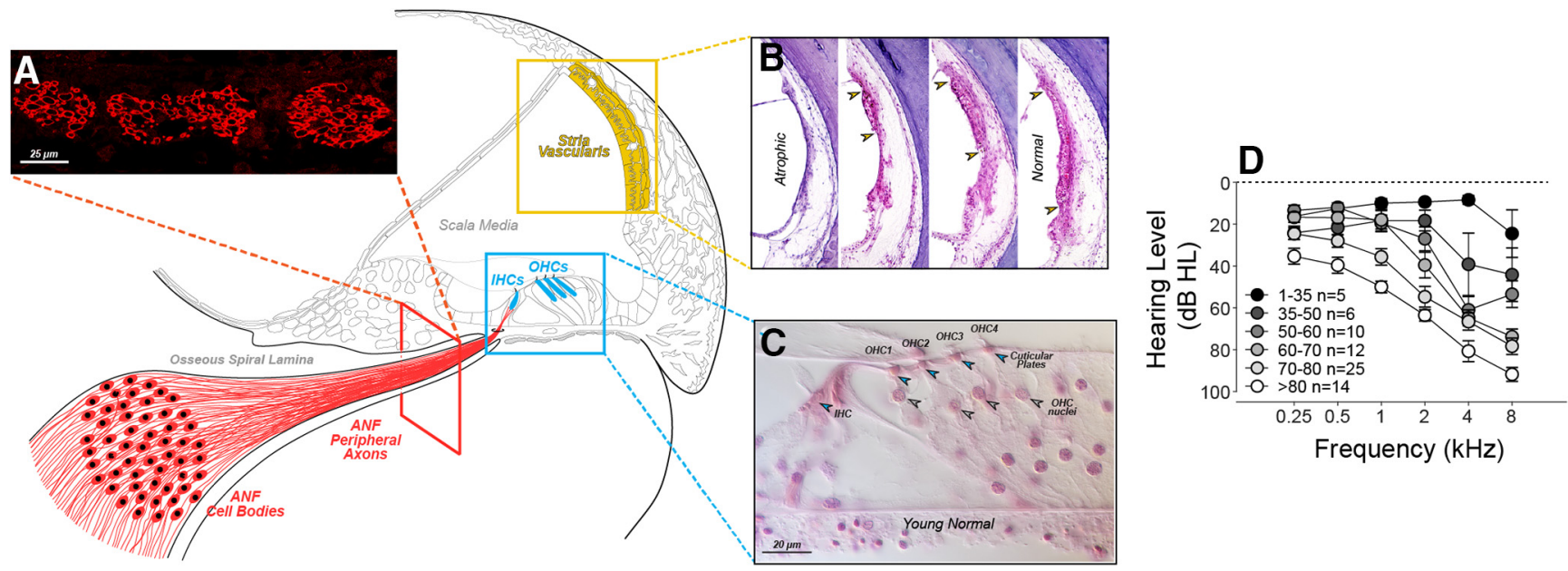

Figure 1. Three histologic features $(\boldsymbol{A}-\boldsymbol{C})$ were quantified as histologic predictors of hearing level $(\boldsymbol{D})$. Peripheral axons of ANFs were counted in cross-sections $(\boldsymbol{A})$. The stria vascularis $(\boldsymbol{B})$, shown from 4 cases (Merchant and Nadol, 2010), was quantified by measuring surviving epithelial area (regions between the arrowheads). IHCs and OHCs ( $\boldsymbol{C}$ were counted using stereocilia and cuticular plates (open arrowheads) as markers of survival (Wu et al., 2019). Audiograms (D) show group mean hearing loss ( \pm SEMs) for all cases analyzed, except 5 that were excluded (from this plot only) because they were chosen for prior study based solely on their unusual audiometric pattern (Pauler et al., 1988). Cases are separated into six age groups as shown in the key. The male (M)/female (F) mix for each group is as follows: age 1-35, $1 \mathrm{M}, 4 \mathrm{~F}$; age 35-50, $5 \mathrm{M}, 1 \mathrm{~F}$; age 50-60, $10 \mathrm{M}, 0 \mathrm{~F}$; age 60-70, $11 \mathrm{M}, 1 \mathrm{~F}$; age 70-80, $17 \mathrm{M}, 8 \mathrm{~F}$, and age $>80,8 \mathrm{M}, 6 \mathrm{~F}$.

(Fig. 1B) is specialized for ion transport (Patuzzi, 2011) and maintains the electric potential that, like a battery, drives current into hair cells when their transduction channels are opened by sound. The outer hair cells (OHCs) also transduce sound into electrical signals but reverse-transduce those electrical signals into mechanical motion, which powers a cochlear amplifier that gives the inner ear its sensitivity (Dallos, 2008). This sensory epithelium is mechanically tuned to high frequencies at the basal end of the spiraling cochlear duct, and to low frequencies at the apex.

Because the inner ear lies deep within the temporal bone, it cannot be biopsied, and its sensory structures cannot be resolved with clinical imaging. Thus, inner-ear pathology has been studied in histologic sections of tissues extracted after death (Schuknecht, 1974). In classic studies (Schuknecht, 1955, 1964, 1974; Schuknecht et al., 1974; Pauler et al., 1988; Ramadan and Schuknecht, 1989; Schuknecht and Gacek, 1993), Schuknecht and colleagues concluded that presbycusis was rarely explained by hair cell loss. Rather, it was associated with strial atrophy and/ or loss of ANFs and often was uncorrelated with any cochlear histopathology (Ramadan and Schuknecht, 1989; Bhatt et al., 2001; Merchant and Nadol, 2010). Correspondingly, studies in aging gerbils also concluded that age-related high-frequency hearing loss is because of strial atrophy, rather than hair cell loss (Tarnowski et al., 1991; Schulte and Schmiedt, 1992; Schmiedt et al., 2002; Dubno et al., 2013).

Understanding the functionally significant structural changes in human presbycusis is critical as therapies for rebuilding a damaged inner ear move to clinical trials (Kujawa and Liberman, 2019), and inclusion criteria are based on audiometric data. Clearly, a therapy to elicit hair cell regeneration would be ineffective in presbycusis if the predominate pathology is strial atrophy. Here, using high-resolution light microscopy (Wu et al., 2020), we provide the first quantitative view of inner ear damage in human presbycusis. By applying rigorous statistical modeling, rather than the case-based approaches of the past, we show that hearing loss is well predicted by hair cell loss, that strial atrophy or auditory-nerve loss contributes little or nothing to the audiometric pattern, and that audiogram slope is not diagnostic for the strial degeneration. (Pauler et al., 1988; Dubno et al., 2013).
We also show that patterns of hair cell loss in aging humans are fundamentally different from those in animal models of aging, and suggest that these differences arise because of the routine ear abuse of life in industrialized societies.

\section{Materials and Methods}

Subjects and groups

Archival slide sets of serially sectioned, celloidin-embedded temporal bones from the Massachusetts Eye and Ear collection were analyzed in the present study. A survey of the entire collection yielded 120 ears that met the following inclusion criteria for this study of "normal aging": (1) adequate preservation for reliable microscopic analysis; (2) no genetically determined or developmental defects; (3) no history of infections, neoplastic growth, vascular disorders, or bone malformation in the ear; (4) no history of otologic surgery; (5) no history of vertigo, dizziness, or sudden sensorineural hearing loss; (6) no autoimmune disease; and (7) no history of exposure to ototoxic drugs. The selected individuals ranged in age from birth to 104 years, and included 75 males and 45 females; all have white, non-Hispanic origins. Of these 120 cases, 77 had audiograms taken within 5.5 years of death, and 54 also had word-recognition scores (expressed as a percentage of monosyllabic, phonetically balanced words correctly identified when presented in quiet at an audible level). All procedures and protocols for the study of human tissues included informed consent and were approved by the Institutional Review Board of the Massachusetts Eye and Ear.

\section{Histologic analysis}

For all histologic analysis, observers were blinded to medical history and audiometric data. For each case, the set of 40-50 archival slides contains $\sim 100$ cuts through the spiraling cochlear duct. These slide sets were used for quantification of hair cell loss and strial atrophy. The cochlear spiral in each case was graphically reconstructed (Merchant and Nadol, 2010) to allow the percent distance along the spiral of each section through the cochlear duct to be computed and converted into frequency $(\mathrm{kHz})$ using a cochlear frequency map for human (Greenwood, 1990), as modified to produce apical-most and basal-most best frequencies of 0.1 and $20 \mathrm{kHz}$, respectively.

Tissue processing. When originally archived, each temporal bone was aldehyde fixed, decalcified, embedded in celloidin, and serially sectioned at $20 \mu \mathrm{m}$ in the horizontal plane. Every 10th section was stained with H\&E and slide-mounted (Merchant and Nadol, 2010), while intervening (unstained) sections were stored in $80 \%$ alcohol. For the present study, selected unstained sections were retrieved, decelloidinized (O'Malley et 


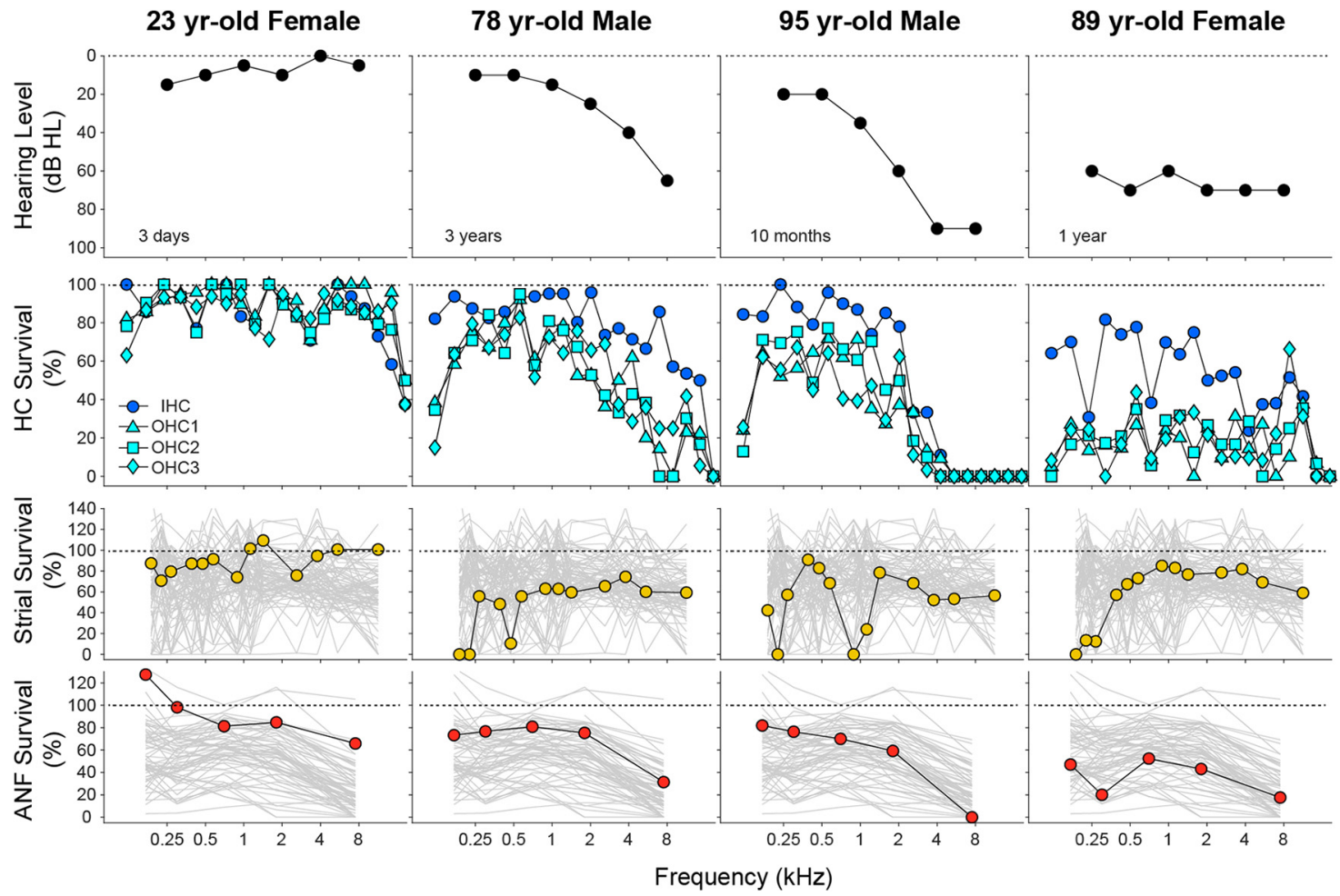

Figure 2. Histopathological analyses from 4 of the normal-aging cases in the present study. For each case, the most recent audiogram is shown in the top row, and the histopathological data for hair cells, stria vascularis, and ANFs are summarized in the second, third, and fourth rows, respectively. Hair cell data are binned into $5 \%$ increments of cochlear length, and the key at the left applies to all columns. Strial and neuronal data are unbinned and are plotted with the profiles from all cases studied (gray). For the histopathological measures, cochlear location has been converted into frequency according to the cochlea frequency map for human (Greenwood, 1990).

al., 2009), and stained with a fluorescent membrane dye (CellMask Orange, Thermo Fisher Scientific, \#C10045) at 1:1000 for 5 min to label myelin sheaths. The sections were then coverslipped with Vectashield.

Hair cell counts. Fractional survival of IHCs and OHCs was quantified in all archival sections from each case using a $100 \times$ oil-immersion objective $(\mathrm{NA}=1.3)$ and differential interference optics on a Nikon EDCCC microscope. In each section through the cochlear duct, the number of remaining cells in each hair cell row was counted, and the number of missing cells was estimated as described in more detail previously (Wu et al., 2020). Dividing the two values yields fractional survival, which needs no further normalization. Hair bundles and cuticular plates were the main criterion for surviving hair cells, rather than cell nuclei, as done in all prior work. The hair cell analysis in each case is based on observations at $\sim 100$ positions along the cochlear spiral.

Peripheral axon counts. Neural degeneration was assessed by counting peripheral axons of ANFs (Fig. 1A), rather than their cell bodies, because cell bodies can survive for years after peripheral axon loss (M. A. Chen et al., 2006; Kujawa and Liberman, 2009; Liu et al., 2015). ANFs were quantified at five locations along the cochlear spiral, one at the tangential section through each half turn, where the axons are cut in cross section and can be precisely counted (Wu et al., 2019). Previously unstained sections were selected from the archive for each half turn, seeking the section near the junction between the limbus and tectorial membrane (Fig. 1). After Cellmask staining (see above), confocal $z$ stacks were acquired with $0.33 \mu \mathrm{m} z$ spacing on an SP8 using a $63 \times$ glycerol objective (NA $=1.3$; Leica Microsystems). Image stacks spanning the top $5 \mu \mathrm{m}$ of the section were acquired. The brightest focal plane was selected, and axons were counted within a $250 \mu \mathrm{m}$ mask. Raw data were normalized to average counts from the appropriate cochlear region in 6 young normal cases, aged 0-5.4 years (Wu et al., 2019).

Strial analysis. Strial atrophy was quantified in the archival slide sets by measuring the cross-sectional area of the stria at 13 cochlear locations, equally spaced at one-eighth of each cochlear turn, avoiding regions where the stria is cut tangentially. At each locus, healthy strial tissue was outlined and measured using computer-aided anatomy software (Neurolucida). The sections were displayed through a video camera connected to a Nikon EDC using a $20 \times$ objective $(\mathrm{NA}=0.75)$. One focal plane, near the middle of the section, was chosen for tracing. Raw data were normalized to average data from the appropriate cochlear region in young normals $(n=5)$, aged $0-5.4$ years.

\section{Statistical modeling}

To select the functionally most important histologic structures and to fit a model, linear regression was performed via the LASSO (Least Absolute Shrinkage and Selection Operator) method using the glmnet package in $\mathrm{R}$ 3.6.1. Before fitting in the LASSO linear regression, the data were preprocessed as follows: (1) histopathological measurements were averaged across audiometric frequency to produce a mean survival and treated as continuous variables; and (2) continuous variables (i.e., histopathological measurements, age, and hearing level) were normalized ( $z$ score). The best model was selected via nested $5 \times 10$-fold cross-validation. The statistical significance of between-group differences, when analyzed as a function of cochlear distance/frequency, was assessed using profile analysis, with the profileR package in $\mathrm{R}$ 3.6.1.

\section{Results}

The present study is based on quantitative histopathological analysis of 120 cases, aged 1-104 years, from the archival temporal bone collection at the Massachusetts Eye and Ear (Merchant et al., 2008). In each case, the fractional survival of IHCs, OHCs, ANFs, and the stria vascularis was computed at numerous locations along the cochlear spiral. Using published maps relating cochlear location to best frequency (Greenwood, 1990), the histopathological data were correlated with the premortem audiometric results from the same individuals, which were available for 77 of the cases, tested within 5.5 years of death. The example cases in Figure 2 illustrate the type of data obtained in each case, as well as a number of general trends in the histopathology. 
Cluster by Stria
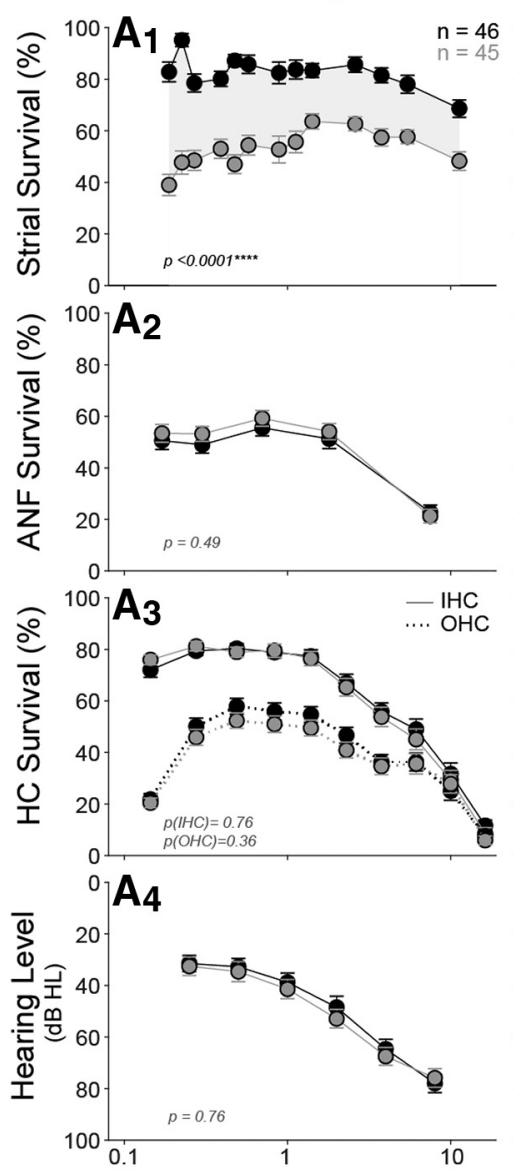

A5

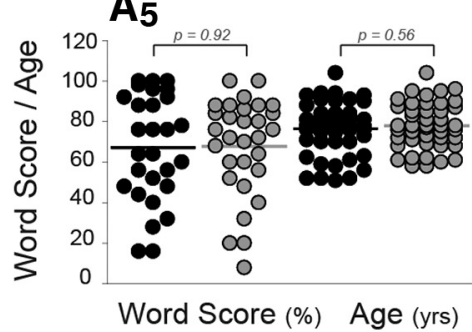

Cluster by ANF

$B_{1}$
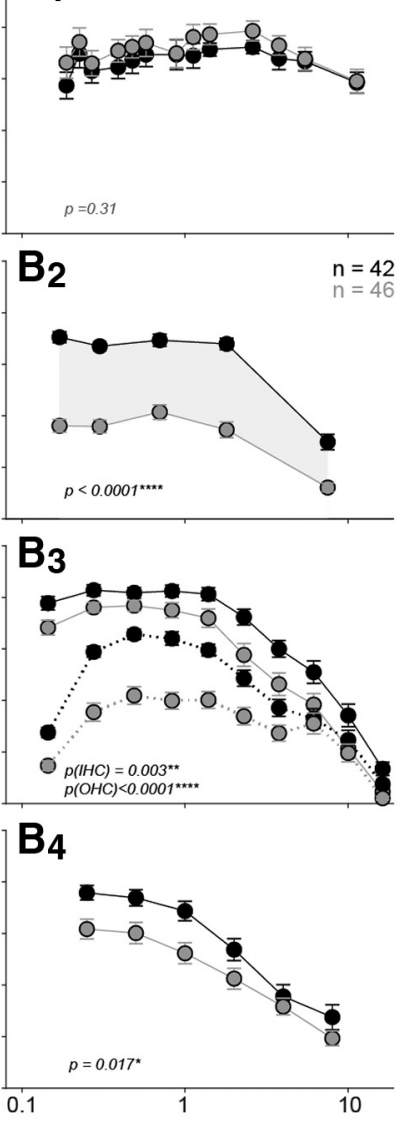

Cluster by $\mathrm{OHC}$

$\mathrm{C}_{1}$
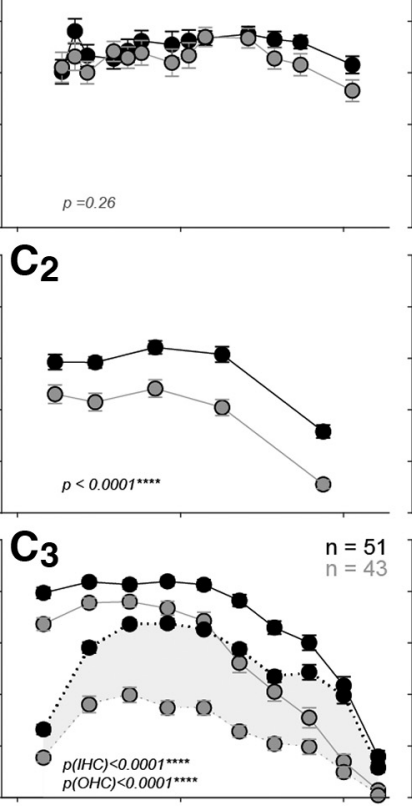

$\mathrm{C}_{4}$

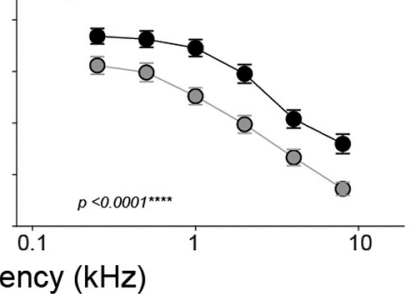

$\mathrm{D}_{1}$

Cluster by IHC
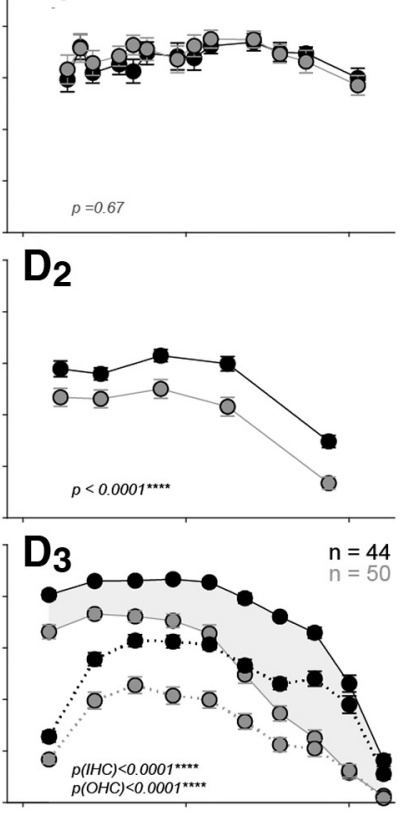

$\mathrm{D}_{4}$

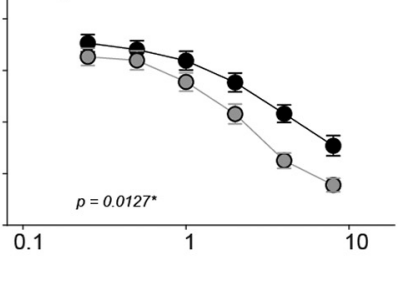

Frequency $(\mathrm{kHz})$

$\mathrm{B}_{5}$

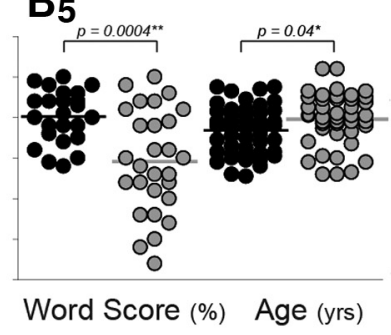

$\mathrm{C}_{5}$

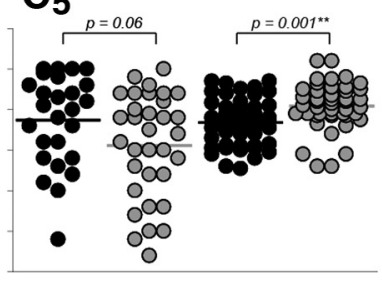

Word Score (\%) Age (yrs)

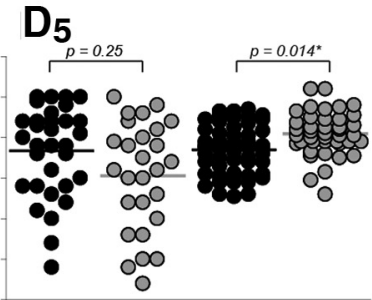

Word Score (\%) Age (yrs)

Figure 3. Cluster analysis shows interactions among predictors and the contribution of each to hearing level and word scores. In each column, $k$-means clustering was performed based on fractional survival of a different histologic metric, as indicated in the header: $\boldsymbol{A}_{1}-\boldsymbol{A}_{5}$, stria; $\boldsymbol{B}_{1}-\boldsymbol{B}_{5}$, ANF; $\boldsymbol{C}_{1}-\boldsymbol{C}_{5}$, OHCs; and $\boldsymbol{D}_{1}-\boldsymbol{D}_{5}$, IHCs. Clustering was adjusted to define two groups of approximately equal size, as shown by the paired functions separated by gray shading ( $n$ values are group sizes). For the top three rows, mean values ( \pm SEMs) are shown for the histologic metric indicated on the $y$ axis at the left. The bottom two rows represent the outcomes for each cluster: means ( \pm SEMs) for audiograms $\left(\boldsymbol{A}_{4}, \boldsymbol{B}_{4}, \boldsymbol{C}_{4}, \boldsymbol{D}_{4}\right)$ and dot plots for word scores $\left(\boldsymbol{A}_{5}, \boldsymbol{B}_{5}\right.$, $\boldsymbol{C}_{5}$, $\left.D_{5}\right)$. Ages are shown in the bottom row, along with statistics for the pairwise comparisons: Student's $t$ test for the bottom row; profile analysis for the top three rows. The dataset for hair cell counts includes all cases older than 50 years $(n=94: 58 \mathrm{M}, 36 \mathrm{~F})$; strial measurements were impossible in 3 cases because of gross portmortem artifact $(n=91: 57 \mathrm{M}, 34 \mathrm{~F})$, and ANF counts were impossible in 6 cases because of faint staining ( $n=88: 57 \mathrm{M}, 31 \mathrm{~F})$.

Although there is age-related hair cell death throughout the cochlea, the IHC loss tends to be greater in the basal (high-frequency) half of the cochlea than the apical half, whereas the apex-base gradient of OHC death is more variable. ANF degeneration is seen throughout the cochlea but is usually worse in the cochlear base. In contrast, strial degeneration is typically worse in the cochlear apex.

To consider these apical-basal gradients more quantitatively, and to probe the interactions among the histopathological metrics, we performed $k$-means clustering on each measure, across all cases $>50$ years old (Fig. 3). The cluster analysis of strial degeneration profiles identified a "good-stria" group and a "bad- stria" group of approximately equal size (Fig. $3 A_{1}$ ). The group means suggest that, when present, strial degeneration tends to be worse in the cochlear apex. In contrast, the mean data derived from ANF or IHC clustering show clear apex-to-base gradients of degeneration (Fig. $3 B_{2}, D_{3}$ ), while OHCs show both apical and basal foci of cell death (Fig. $3 C_{3}$ ).

Contrary to prevailing views (Pauler et al., 1988; Merchant and Nadol, 2010), the two strial clusters did not differ significantly with respect to hearing loss (Fig. $3 A_{4} ; p=0.764$ by profile analysis), and the audiometric slope in the "bad-stria" group was not flat, as has been suggested (Pauler et al., 1988). Indeed, the cluster analysis suggests that strial condition is uncorrelated with 

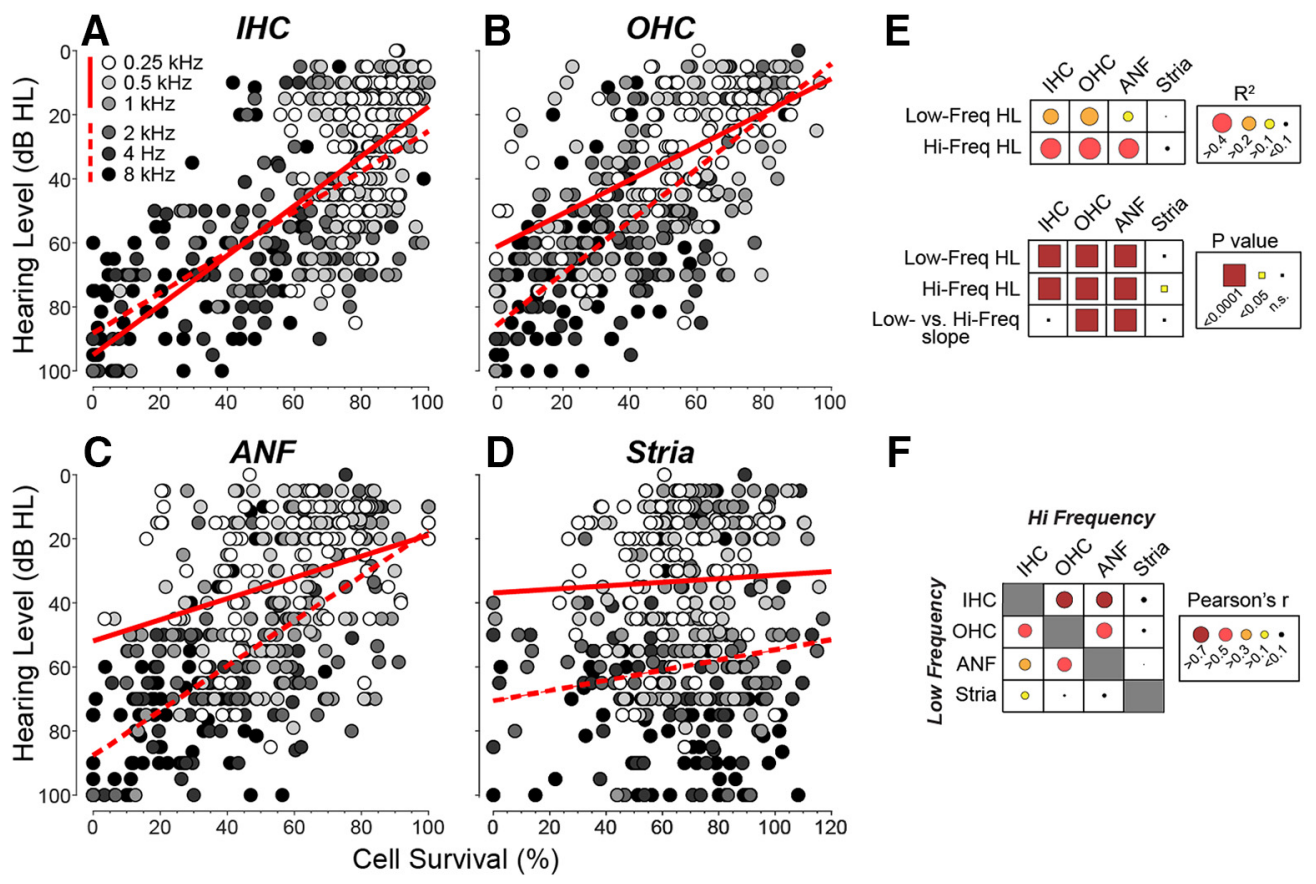

Figure 4. Regression analyses of the four histologic predictors of hearing level $(\boldsymbol{A}-\boldsymbol{E})$, and the pairwise correlations among them $(\boldsymbol{F})$, show that strial atrophy is relatively independent of the other three metrics and is poorly predictive of threshold. Each point in each scatterplot represents the mean of the relevant metric, binned over cochlear loci appropriate to the audiometric frequency, as coded in grayscale (key in $\boldsymbol{A}$ also applies to $\boldsymbol{B}-\boldsymbol{D})$. The regression was assessed separately for low $(0.25,0.5$, and 1.0$)$ versus high $(2.0,4.0$, and 8.0$)$ frequencies, and the best-fit straight lines are shown by solid and dashed lines, respectively. The $r^{2}$ values and $p$ values for the least-squares regression are indicated graphically in $\boldsymbol{E}$, along with $p$ values for the significance of the difference of slope between low- and high-frequency regressions. The Pearson's $r$ values for the pairwise correlations between each of the histologic predictors are indicated graphically in $\boldsymbol{F}$ : the raw scatterplots are in Figure 5 . Hair cell measures were completed in 77 cases ( $43 \mathrm{M}, 34 \mathrm{~F})$, ANF measure in 72 cases $(39 \mathrm{M}, 33 \mathrm{~F})$, and strial measures in 76 cases ( $43 \mathrm{M}, 33 \mathrm{~F})$.

any of the other measures: that is, with IHC or OHC loss (Fig. $3 A_{3}$ ), neural loss (Fig. $3 A_{2}$ ), word-recognition scores or age (Fig. $\left.3 A_{5}\right)$.

In contrast, the analyses of ANFs, IHCs, and OHCs show significant intercluster differences in audiometric thresholds (Fig. $3 B_{4}, C_{4}, D_{4}$ ), as well as age (Fig. $3 B_{5}, C_{5}, D_{5}$ ). The largest audiometric differences are seen when clustering is based on OHCs (Fig. $3 C_{4}$ ), as expected given the role of these cells in powering the cochlear amplifier needed for hearing sensitivity (Dallos, 2008). The threshold differences between the ANF clusters (Fig. $3 B_{4}$ ), especially at low frequencies, is surprising since neural degeneration, per se, should not strongly affect audiometric thresholds (Schuknecht and Woellner, 1955). This association likely reflects the strong correlation between ANF degeneration and OHC loss (see below). The only significant differences in word scores are seen when clustering for ANF degeneration (Fig. $3 B_{5}$ ), which fits with the hypothesized importance of neural density in discrimination of complex acoustic signals, rather than in signal detection in quiet (Wu et al., 2019).

To more directly identify the functionally important structural changes underlying age-related hearing loss, we averaged each histopathological measure, in each case, in the region surrounding each audiometric measure. Plotting each of the four histopathological predictors versus hearing level for all cases (Fig. $4 A-D$ ) shows that, when only one predictor is used in the regression, hearing level is best predicted by OHC survival in both the high- and low-frequency halves of the hearing range (see $r^{2}$ values in Fig. 4E). However, in the high-frequency half of the cochlea, the single-variable predictions for IHCs and ANFs are very close to those for OHCs. The slope of the relation between OHCs and hearing level (Fig. $4 B$ ) is steeper at high frequencies, as expected based on the larger contribution of the OHC-based cochlear amplifier at high frequencies (Liberman et al., 2002; Schmiedt et al., 2002). As expected based on the cluster analysis, the contributions of strial atrophy to hearing level are insignificant in both the high- and low-frequency regions (Fig. $4 D, E)$.

Parsing the relative contributions of ANF, IHC, and OHC survival to hearing loss is complicated by the collinearity among the various histopathological measures (Pearson's $r$ values for pairwise correlations are represented graphically in Fig. 4F; scatterplots are shown in Fig. 5). To deal with these collinearities, we adjusted the joint effect of histopathological predictors, plus age, into a multivariable linear regression model using the LASSO method (Yang and Zou, 2015). Recognizing the important differences between the apical and basal halves of the cochlea, for example, with respect to the contribution of OHCs to cochlear amplification (Schmiedt et al., 2002), we divided the six audiometric frequencies into low-frequency and high-frequency halves, and fit separate regression models for each (Fig. 6). All the predictors were normalized to the same scale; thus, the absolute values of the LASSO coefficients (Fig. 6B) indicate the relative importance of each predictor to its respective model. In all, $65.3 \%$ of the variance in hearing level is explained by the model (Fig. 6A). In both high- and low-frequency regions, OHC and IHC survival are the most important histologic predictors (Fig. $6 B$ ). ANF survival contributes to the prediction of hearing loss only in the basal half of the cochlea, and strial condition does not contribute in either cochlear region. The fact that age improves the predictive power of both models indicates there are important age-related effects that have not been captured by the present histopathological analysis of the cochlea. These uncaptured 

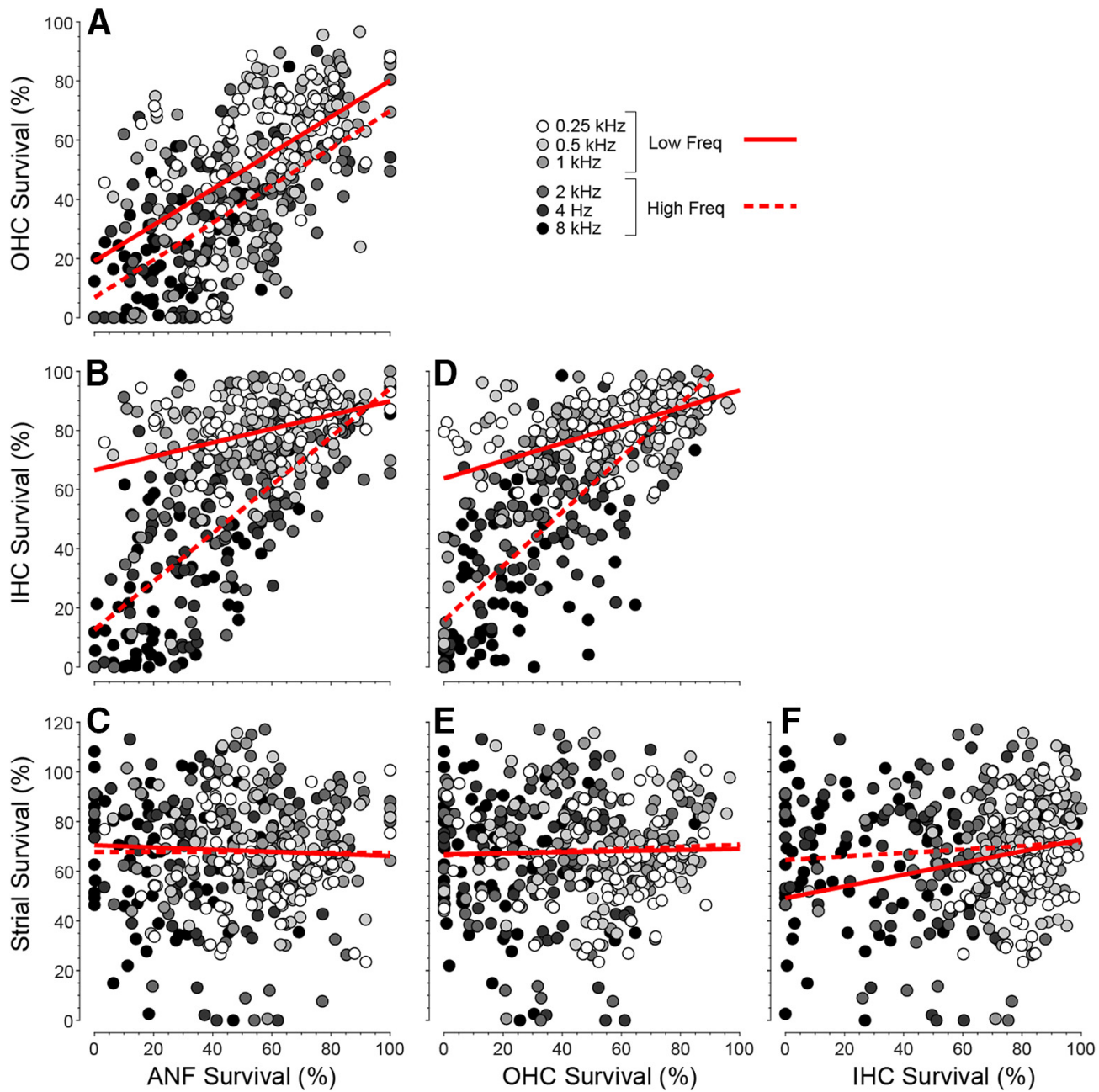

Figure 5. A-F, Pairwise correlations among the histologic predictors. As in Figure 4, the histologic data in each case are binned and averaged over the cochlear locations appropriate to each audiometric frequency: thus, each case produces 6 points on each graph. Data were derived from the 71 cases $(39 \mathrm{M}, 32 \mathrm{~F})$ with recent audiograms and complete histologic data. Leastsquares best-fit straight lines are separately computed for low- versus high-frequency regions (solid vs dashed lines).

effects could be subtler pathologies in surviving structures (e.g., damage to the hair cell stereocilia) (Liberman and Dodds, 1984) or changes in the central auditory pathways outside the scope of the present investigation.

\section{Discussion}

Differences from prior studies of human presbycusis

Most forms of acquired hearing loss, including presbycusis, arise in the inner ear, but understanding the underlying damage is limited by the inability to biopsy the inner ear or to reveal cellular detail noninvasively. Prior studies of human otopathology (for review, see Merchant and Nadol, 2010) have been case-based, and the relations between histopathology and hearing loss have rarely been quantitatively examined. A rigorous understanding of these relationships is increasingly important, as biological therapeutics for inner ear
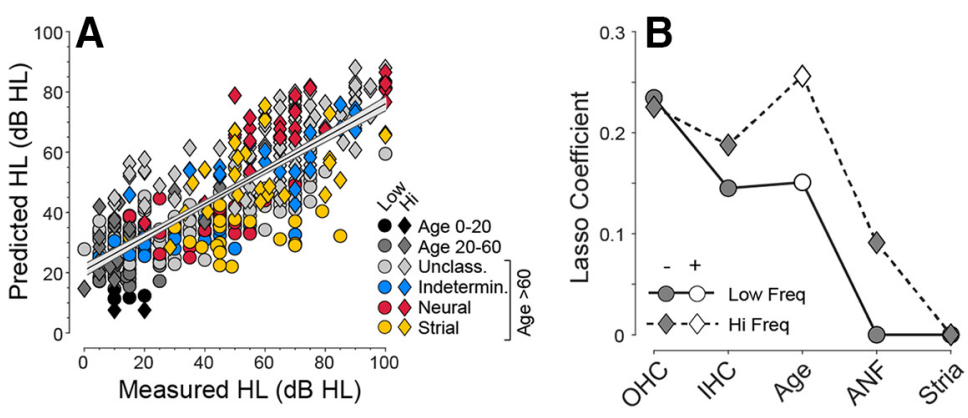

Figure 6. Multivariable LASSO regression was used to derive the best weightings (coefficients) of the histologic metrics (plus age; $\boldsymbol{B}$ ) should be used to predict hearing level $(\boldsymbol{A})$. As for Figure 4, the regression analysis was separately conducted for low- versus high-frequency regions (circles vs diamonds in $\boldsymbol{A}$ and $\boldsymbol{B}$ ). The regression line, plus $95 \% \mathrm{Cl}$, is shown for both frequency regions combined. The differences in coefficient polarity $(\boldsymbol{B})$ arise because hearing level is expressed as a loss and is positively correlated with age, while histologic metrics are expressed as survival and are negatively correlated with hearing level. The data are derived from the 71 cases (39 M, 32 F) with recent audiograms ( $<5.5$ years) and complete histologic data. The younger ears ( $<60$ years) are grouped as shown in key (age 0-20, 0 M, $1 \mathrm{~F}$; age 20-60, $6 \mathrm{M}, 4$ F; unclassified, 25 M, 11 F; indeterminate, 5 M, 3 F; neural, 1 M, 7 F; strial, 2 M, 6 F). The older ears ( $\geq 60$ years) comprise all the exemplars of the four presbycusis types previously studied ( $n=24$ of 35) (Schuknecht and Gacek, 1993; Merchant and Nadol, 2010), except 11 that were excluded (from this plot only) because ANF counts were impossible or recent audiograms ( $<5.5$ years) were unavailable. The unclassified group comprises additional normal-aging individuals from the Massachusetts Eye and Ear collection not previously studied. 

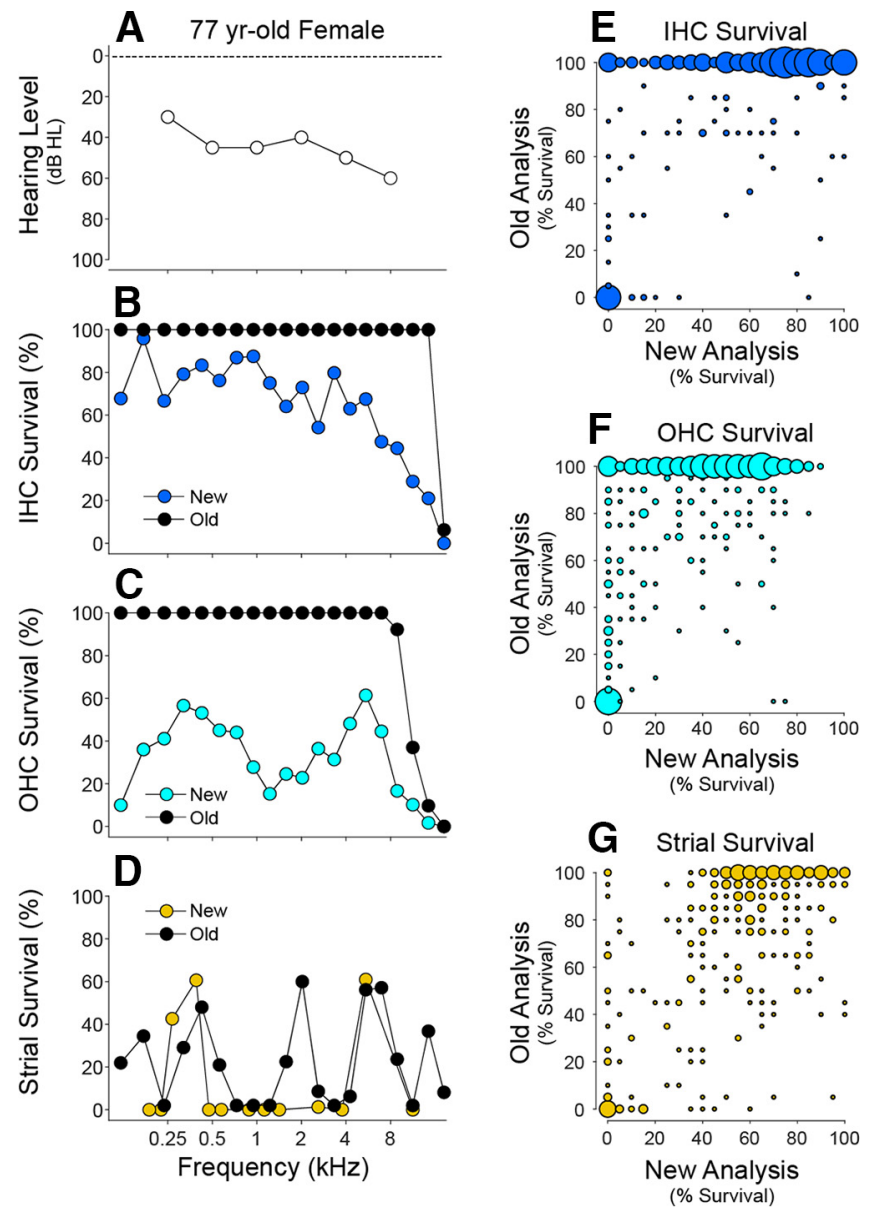

Figure 7. Comparison of hair cell and strial survival metrics from the same slide sets: published data, that is, "Old analysis" (Schuknecht and Gacek, 1993) versus the "New Analysis" in the present study. $\boldsymbol{A}-\boldsymbol{D}$, One example case, including the audiogram $(\boldsymbol{A})$ and a comparison of results for $\mathrm{IHCS}(\boldsymbol{B}), 0 \mathrm{HCS}(\boldsymbol{C})$, and stria $(\boldsymbol{D})$. $\boldsymbol{E}-\boldsymbol{G}$, Summary comparisons from all the exemplar cases $(n=35)$ from the presbycusis studies of Schuknecht and colleagues (Schuknecht and Gacek, 1993; Merchant and Nadol, 2010). To create the summary plots in $\boldsymbol{E}-\boldsymbol{G}$, we scanned the published graphs, digitized and extracted the histogram values, and converted the data into mean survival for each cell type, within each 5\% cochlear-length bin. For the same cases, we computed the mean survival for each cell type over the same $5 \%$ length bin as assessed in the present study. To display the results, we rounded each mean value to the nearest $5 \%$ survival bin, and created these scatterplots in which the area of the symbol is proportional to the number of observations falling within each bin of the matrix.

disorders are on the horizon, and subject-inclusion criteria depend on predicting the degree and pattern of hair cell or neural loss from noninvasive tests of hearing function.

Almost all human presbycusis studies are based on light-microscopic evaluation of semiserial temporal-bone sections. However, prior studies have "binarized" the hair cell analysis, rating cell survival in each section as either $0 \%$ or $100 \%$ (Ramadan and Schuknecht, 1989; Schuknecht and Gacek, 1993; Nelson and Hinojosa, 2006; Merchant and Nadol, 2010; Landegger et al., 2016; Linthicum et al., 2017). Using high-power DIC objectives to optically section the slides, we assessed fractional survival of hair cells (Wu et al., 2020), and conclusions from the two approaches dramatically differ (Fig. $7 B, C$ ). In a binarized analysis, only complete hair cell loss is noted (Fig. 7E, $F)$. Thus, Schuknecht's influential studies of presbycusis (Schuknecht, 1955, 1964, 1974; Pauler et al., 1988; Ramadan and Schuknecht, 1989; Schuknecht and Gacek, 1993) concluded that hair cell loss rarely explains the audiometric pattern, and that strial degeneration or neuronal loss is more often responsible.

Cases analyzed here included all 35 exemplars from Schuknecht's presbycusis studies (Schuknecht, 1955, 1964, 1974; Schuknecht et al., 1974; Pauler et al., 1988; Ramadan and

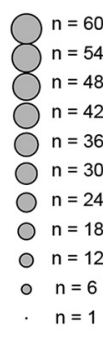

Schuknecht, 1989; Schuknecht and Gacek, 1993; Merchant and Nadol, 2010): the original slide sets are still available from the Massachusetts Eye and Ear collection. In our multivariable regression analysis of audiogram prediction, coding the data according to Schuknecht's presbycusis classification, that is, sensory, strial, neural, or indeterminate (Schuknecht and Gacek, 1993; Merchant and Nadol, 2010), suggests that that all four presbycusis "types" are equally well "explained" by the same weighting of $\mathrm{OHC}, \mathrm{IHC}$, and neural losses (Fig. 6A); thus, that this classic, and widely cited, typology is not informative of the underlying causes of agerelated hearing loss.

\section{Inner ear pathology and audiometric slopes: metabolic versus sensory presbycusis}

The gold-standard hearing test for humans is the audiogram. Animal studies show that thresholds provide a sensitive measure of $\mathrm{OHC}$ function because OHCs are biological amplifiers, enhancing the sound-induced vibrations of the sensory epithelium (Dallos, 2008). Consistent with this, OHC survival was the most important histopathogical predictor of thresholds in the present analysis (Fig. 6B). IHCs are also critical for hearing because myelinated ANFs contact only IHCs (Spoendlin, 1972; Liberman, 1982). However, animal studies suggest that IHC loss, per se, does not affect thresholds until the loss exceeds 80\% (Lobarinas et al., 2013) because thresholds are tested at octavefrequency intervals, and the normal IHC density of 500 cells per octave provides massive redundancy (Wu et al., 2019). Consistent with this, IHC survival was less important than OHC survival as a threshold predictor (Fig. 6B). Although we need ANFs to hear, thinning the ANF population, without hair cell loss, does not elevate thresholds (Schuknecht and Woellner, 1955). Therefore, a minor role of ANF survival in hearing-level prediction (Fig. 6B) was expected. As the neural loss increases, it degrades the representation of complex stimuli in central pathways; thus, only ANF survival was significantly correlated with word-recognition scores (Fig. $3 B_{5}$ ).

Strial atrophy was common in the aging ears we studied: in atrophic cases, the strial epithelium was reduced by almost $50 \%$ across the entire cochlea (Fig. $3 A_{1}$ ). The poor correlation between strial damage and either hair cell or neural survival (Figs. $4 F, 5)$ suggests that a chronic decrease in endolymphatic polarization does not jeopardize either hair cell or neural health. Nevertheless, the prevalence of strial atrophy presents a challenge for therapeutics designed to restore hearing: regenerating hair cells will not restore normal thresholds to ears with strial atrophy, and present results suggest no clear-cut way to diagnose the degree of strial damage. 
A decrease in endolymphatic potential should raise thresholds, as demonstrated in aging gerbils (Schmiedt et al., 2002). Surprisingly, strial pathology was uncorrelated with thresholds in our study (Figs. $3 A_{4}, 4 D$ ) and was not useful in hearing-level prediction (Fig. 6). Apparently, by the time there is significant strial atrophy, there is already so much OHC loss (Fig. $3 A_{3}$ ) that the cochlear amplifier is dysfunctional. Without the amplifier, a reduction of endolymphatic potential from, for example, $100-50 \mathrm{mV}$, reduces the driving force for IHC transduction currents from 160 to $110 \mathrm{mV}$ (IHC intracellular potential is $-60 \mathrm{mV}$ ) (Fettiplace, 2017). In a linear regimen (without cochlear amplification), this decreases receptor currents by only 50 of 160 (i.e., $3.25 \mathrm{~dB}$ ), which is audiologically insignificant.

Prior human and animal studies suggested that two types of presbycusis, that is, "metabolic" (because of strial atrophy) versus "sensory" (because of hair cell loss), can be diagnosed by audiogram slope: that is, shallow down-sloping (metabolic) versus steeply down-sloping (sensory) (Pauler et al., 1988; Schmiedt et al., 2002; Dubno et al., 2013). Our analysis showed no effect of strial atrophy on slope (Fig. $3 A_{4}$ ), rather that audiogram slope is determined by apical-basal gradients of IHC and OHC loss (Figs. $2,6 B)$. The idea that sensory cell losses lead to steeply sloping audiograms (Schuknecht and Gacek, 1993) arose from binarization of hair cell counts: if only complete hair cell loss is noted, the associated profound hearing loss will necessarily produce a precipitous drop in the audiogram (e.g., Fig. 2C). Pure strial atrophy will likely result in a gently down-sloping audiogram, and pure strial atrophy may be the dominant phenotype of the aging gerbil (Schmiedt et al., 2002); however, a pure strial phenotype is rarely seen in human presbycusis.

\section{Human presbycusis versus animal models of age-related hearing loss}

Age-related hearing impairment in humans is extensively documented (e.g., Gordon-Salant, 2005), and average audiograms for our cases (Fig. $8 B_{1}$ ) are within $10 \mathrm{~dB}$ of age-matched norms (Rosen et al., 1962). Many laboratories have studied cochlear dysfunction and histopathology in aging animals (Ulehlova, 1975; Bhattacharyya and Dayal, 1985; Tarnowski et al., 1991; G. D. Chen et al., 2009; Ohlemiller et al., 2010; Cai et al., 2018); however, without quantitative histopathological norms for aging humans, it has been impossible to judge which aging animal is most relevant. Several mammals (rats, mice, and gerbils) show an apical focus of $\mathrm{OHC}$ loss (Fig. $8 A_{3}$ ), similar to that in humans (Fig. $8 B_{3}$ ). This aspect of human otopathology has been overlooked because the loss is fractional, and fractional loss has been ignored. Furthermore, apical hair cell loss is not reflected in the audiogram, because (1) the apex is tuned to lower frequencies than those tested (Greenwood, 1990), and (2) OHCs contribute less cochlear amplification at low frequencies (Liberman et al., 2002). Nevertheless, the resultant cochlear dysfunction likely contributes to impairment of speech perception, for which lowfrequency information is critical.

Humans have more mid- and high-frequency threshold shift (Fig. $8 B_{1}$ ) than animals (Fig. $8 A_{1}$ ) and, correspondingly, show more hair cell loss (Fig. $8 B_{2}, B_{3}$ vs Fig. $8 A_{2}, A_{3}$ ). The contrast is most dramatic for best-studied model, the aging gerbil (Tarnowski et al., 1991) (Fig. 8A 3 ). In gerbil, apical hair cell loss may explain the low-frequency threshold shifts, but high-frequency losses (Fig. $8 A_{1}$ ) have been convincingly correlated with strial degeneration (Gratton et al., 1996; Schmiedt et al., 2002): the associated reduction in endolymphatic potential has been measured, and the threshold elevations are quantitatively appropriate (Schmiedt et al., 2002). This comprehensive body of animal work has reinforced the idea that presbycusis is not sensory, but metabolic (Dubno et al., 2013). Although these ideas fit with Schuknecht's typology, the gerbil patterns are not representative of aging humans (Fig. 8). Indeed, none of the animal models 
mimics the massive IHC or OHC loss in the basal half of the cochlea, documented here for the first time in humans.

\section{Age-related hearing loss versus noise-induced hearing loss}

Animal studies show that the first signs of permanent noiseinduced hearing loss are typically at high frequencies, regardless of the frequency spectrum of the noise exposure (Liberman and Kiang, 1978). For decades, researchers have wondered whether age-related high-frequency hearing loss is an avoidable result of daily ear abuse from the ubiquitous noise of industrialized society. Classic audiometric studies of aged individuals from isolated societal groups supported this idea (e.g., by showing $60 \mathrm{~dB}$ less high-frequency hearing loss in aging Sudanese tribesmen than in age-matched Americans) (Rosen et al., 1962). Other studies have reached similar conclusions with experimental designs that controlled for genetic factors (Goycoolea et al., 1986).

One obvious difference between animal and human studies is that laboratory animals are born and raised in highly controlled acoustic environments. To gain insight into the role of acoustic overexposure in age-related cochlear histopathology in humans, we separated our normal-aging cohort into those with versus without a noise-exposure history (i.e., occupational exposures in a noisy workplace or in the military). Although the noise metric was relatively crude, our noise-history group showed significantly more hair cell loss, especially in the basal half of the cochlea, where the differences with animal data are also greatest (Fig. $8 B 2, B 3)$. This additional loss in the noise group was most striking for OHCs, and especially among the 50- to 75-year-old group. This, along with the animal data, is consistent with the idea that the apical focus of hair cell damage is inherently agerelated, whereas the larger, and more functionally significant, basal loss in humans is largely noise-induced. If true, the bad news is that we are all abusing our ears, to our significant functional detriment, as we age. The good news is that raised consciousness about the dangers of noisy environments could lead to an improved hearing prognosis for the aging population.

\section{References}

Arrington LR, Beaty TC Jr, Kelley KC (1973) Growth, longevity, and reproductive life of the Mongolian gerbil. Lab Anim Sci 23:262-265.

Bhatt KA, Liberman MC, Nadol JB Jr (2001) Morphometric analysis of agerelated changes in the human basilar membrane. Ann Otol Rhinol Laryngol 110:1147-1153.

Bhattacharyya TK, Dayal VS (1985) Age-related cochlear hair cell loss in the chinchilla. Ann Otol Rhinol Laryngol 94:75-80.

Bielefeld EC, Coling D, Chen GD, Li M, Tanaka C, Hu BH, Henderson D (2008) Age-related hearing loss in the Fischer 344/NHsd rat substrain. Hear Res 241:26-33.

Bohne BA, Gruner MM, Harding GW (1990) Morphological correlates of aging in the chinchilla cochlea. Hear Res 48:79-91.

Cai R, Montgomery SC, Graves KA, Caspary DM, Cox BC (2018) The FBN rat model of aging: investigation of $A B R$ waveforms and ribbon synapse changes. Neurobiol Aging 62:53-63.

Chen GD, Li M, Tanaka C, Bielefeld EC, Hu BH, Kermany MH, Salvi R, Henderson D (2009) Aging outer hair cells (OHCs) in the Fischer 344 rat cochlea: function and morphology. Hear Res 248:39-47.

Chen MA, Webster P, Yang E, Linthicum FH Jr (2006) Presbycusic neuritic degeneration within the osseous spiral lamina. Otol Neurotol 27:316322.

Dallos P (2008) Cochlear amplification, outer hair cells and prestin. Curr Opin Neurobiol 18:370-376.

Dubno JR, Eckert MA, Lee FS, Matthews LJ, Schmiedt RA (2013) Classifying human audiometric phenotypes of age-related hearing loss from animal models. J Assoc Res Otolaryngol 14:687-701.

Fettiplace R (2017) Hair cell transduction, tuning, and synaptic transmission in the mammalian cochlea. Compr Physiol 7:1197-1227.
Fischer N, Johnson Chacko L, Glueckert R, Schrott-Fischer A (2020) Age-dependent changes in the cochlea. Gerontology 66:33-37.

Fox RR, Witham BA, Neleski LA (1997) Handbook of genetically standardized JAX mice. Bar Harbor, ME: Jackson Laboratory.

Goman AM, Lin FR (2018) Hearing loss in older adults: from epidemiological insights to national initiatives. Hear Res 369:29-32.

Gordon-Salant S (2005) Hearing loss and aging: new research findings and clinical implications. J Rehabil Res Dev 42:9-24.

Goycoolea MV, Goycoolea HG, Farfan CR, Rodriguez LG, Martinez GC, Vidal R (1986) Effect of life in industrialized societies on hearing in natives of Easter Island. Laryngoscope 96:1391-1396.

Gratton MA, Schmiedt RA, Schulte BA (1996) Age-related decreases in endocochlear potential are associated with vascular abnormalities in the stria vascularis. Hear Res 94:116-124.

Greenwood DD (1990) A cochlear frequency-position function for several species: 29 years later. J Acoust Soc Am 87:2592-2605.

Keithley EM (2019) Pathology and mechanisms of cochlear aging. J Neurosci Res. Advance online publication. Retrieved May 7, 2019. doi: 10.1002/ jnr.24439.

Kujawa SG, Liberman MC (2009) Adding insult to injury: cochlear nerve degeneration after "temporary" noise-induced hearing loss. J Neurosci 29:14077-14085.

Kujawa SG, Liberman MC (2019) Translating animal models to human therapeutics in noise-induced and age-related hearing loss. Hear Res 377:4452.

Landegger LD, Psaltis D, Stankovic KM (2016) Human audiometric thresholds do not predict specific cellular damage in the inner ear. Hear Res 335:83-93.

Liberman MC (1982) Single-neuron labeling in the cat auditory nerve. Science 216:1239-1241.

Liberman MC, Dodds LW (1984) Single-neuron labeling and chronic cochlear pathology: III. Stereocilia damage and alterations of threshold tuning curves. Hear Res 16:55-74.

Liberman MC, Kiang NY (1978) Acoustic trauma in cats: cochlear pathology and auditory-nerve activity. Acta Otolaryngol Suppl 358:1-63.

Liberman MC, Gao J, He DZ, Wu X, Jia S, Zuo J (2002) Prestin is required for electromotility of the outer hair cell and for the cochlear amplifier. Nature 419:300-304.

Linthicum FH Jr, Doherty JK, Lopez IA, Ishiyama A (2017) Cochlear implant histopathology. World J Otorhinolaryngol Head Neck Surg 3:211-213.

Lipman RD, Chrisp CE, Hazzard DG, Bronson RT (1996) Pathologic characterization of brown Norway, brown Norway x Fischer 344, and Fischer $344 \mathrm{x}$ brown Norway rats with relation to age. J Gerontol A Biol Sci Med Sci 51:B54-B59.

Liu W, Edin F, Atturo F, Rieger G, Lowenheim H, Senn P, Blumer M, Schrott-Fischer A, Rask-Andersen H, Glueckert R (2015) The pre- and post-somatic segments of the human type I spiral ganglion neurons: structural and functional considerations related to cochlear implantation. Neuroscience 284:470-482.

Lobarinas E, Salvi R, Ding D (2013) Insensitivity of the audiogram to carboplatin induced inner hair cell loss in chinchillas. Hear Res 302:113-120.

Merchant SN, Nadol JB (2010) Schuknecht's pathology of the ear, Ed 3. Shelton, CT: People's Medical Publishing House.

Merchant SN, McKenna MJ, Adams JC, Nadol JB Jr, Fayad J, Gellibolian R, Linthicum FH Jr, Ishiyama A, Lopez I, Ishiyama G, Baloh R, Platt C (2008) Human temporal bone consortium for research resource enhancement. J Assoc Res Otolaryngol 9:1-4.

Nelson EG, Hinojosa R (2006) Presbycusis: a human temporal bone study of individuals with downward sloping audiometric patterns of hearing loss and review of the literature. Laryngoscope 116:1-12.

Nowal RN (2018) Walker's mammals of the world. Baltimore: Johns Hopkins University.

Ohlemiller KK, Dahl AR, Gagnon PM (2010) Divergent aging characteristics in $\mathrm{CBA} / \mathrm{J}$ and $\mathrm{CBA} / \mathrm{CaJ}$ mouse cochleae. J Assoc Res Otolaryngol 11:605623.

O’Malley JT, Burgess BJ, Jones DD, Adams JC, Merchant SN (2009) Techniques of celloidin removal from temporal bone sections. Ann Otol Rhinol Laryngol 118:435-441.

Patuzzi R (2011) Ion flow in stria vascularis and the production and regulation of cochlear endolymph and the endolymphatic potential. Hear Res 277:4-19. 
Pauler M, Schuknecht HF, White JA (1988) Atrophy of the stria vascularis as a cause of sensorineural hearing loss. Laryngoscope 98:754-759.

Ramadan HH, Schuknecht HF (1989) Is there a conductive type of presbycusis? Otolaryngol Head Neck Surg 100:30-34.

Rosen S, Bergman M, Plester D, El-Mofty A, Satti MH (1962) Presbycusis study of a relatively noise-free population in the Sudan. Ann Otol Rhinol Laryngol 71:727-743.

Schmiedt RA, Lang H, Okamura HO, Schulte BA (2002) Effects of furosemide applied chronically to the round window: a model of metabolic presbyacusis. J Neurosci 22:9643-9650.

Schuknecht HF (1955) Presbycusis. Laryngoscope 65:402-419.

Schuknecht HF (1964) Further observations on the pathology of presbycusis. Arch Otolaryngol 80:369-382.

Schuknecht HF (1974) Pathology of the ear. Cambridge, MA: Harvard UP.

Schuknecht HF, Gacek MR (1993) Cochlear pathology in presbycusis. Ann Otol Rhinol Laryngol 102:1-16.

Schuknecht HF, Woellner RC (1955) An experimental and clinical study of deafness from lesions of the cochlear nerve. J Laryngol Otol 69:75-97.

Schuknecht HF, Watanuki K, Takahashi T, Belal AA Jr, Kimura RS, Jones DD, Ota CY (1974) Atrophy of the stria vascularis, a common cause for hearing loss. Laryngoscope 84:1777-1821.

Schulte BA, Schmiedt RA (1992) Lateral wall Na,K-ATPase and endocochlear potentials decline with age in quiet-reared gerbils. Hear Res 61:35-46.

Solleveld HA, Haseman JK, McConnell EE (1984) Natural history of body weight gain, survival, and neoplasia in the F344 rat. J Natl Cancer Inst 72:929-940.
Spoendlin H (1972) Innervation densities of the cochlea. Acta Otolaryngol 73:235-248.

Tarnowski BI, Schmiedt RA, Hellstrom LI, Lee FS, Adams JC (1991) Agerelated changes in cochleas of mongolian gerbils. Hear Res 54:123-134.

Tawfik KO, Klepper K, Saliba J, Friedman RA (2019) Advances in understanding of presbycusis. J Neurosci Res. Advance online publication. Retrieved Apr 4, 2019. doi: 10.1002/jnr.24426.

Turner JG, Caspary DM (2005) Comparison of two rat strains of aging: peripheral pathology and GABA changes in the inferior colliculus. In: Auditory plasticity (Syka J, Merzenich M, eds), pp 217-225. Prague Symposium Proceedings.

Ulehlova L (1975) Ageing and the loss of auditory neuroepithelium in the guinea pig. Adv Exp Med Biol 53:257-264.

Wu PZ, Liberman LD, Bennett K, de Gruttola V, O’Malley JT, Liberman MC (2019) Primary neural degeneration in the human cochlea: evidence for hidden hearing loss in the aging ear. Neuroscience 407:8-20.

Wu PZ, Wen WP, O’Malley JT, Liberman MC (2020) Assessing fractional hair cell survival in archival human temporal bones. Laryngoscope 130:487-495.

Yang Y, Zou H (2015) A fast unified algorithm for solving group-lasso penalized learning problems. Stat Comput 25:1129-1141.

Yuan R, Meng Q, Nautiyal J, Flurkey K, Tsaih SW, Krier R, Parker MG, Harrison DE, Paigen B (2012) Genetic coregulation of age of female sexual maturation and lifespan through circulating IGF1 among inbred mouse strains. Proc Natl Acad Sci USA 109:8224-8229. 